\title{
Expediting evidence synthesis for healthcare decision-making: Exploring attitudes and perceptions towards rapid reviews using Q methodology
}

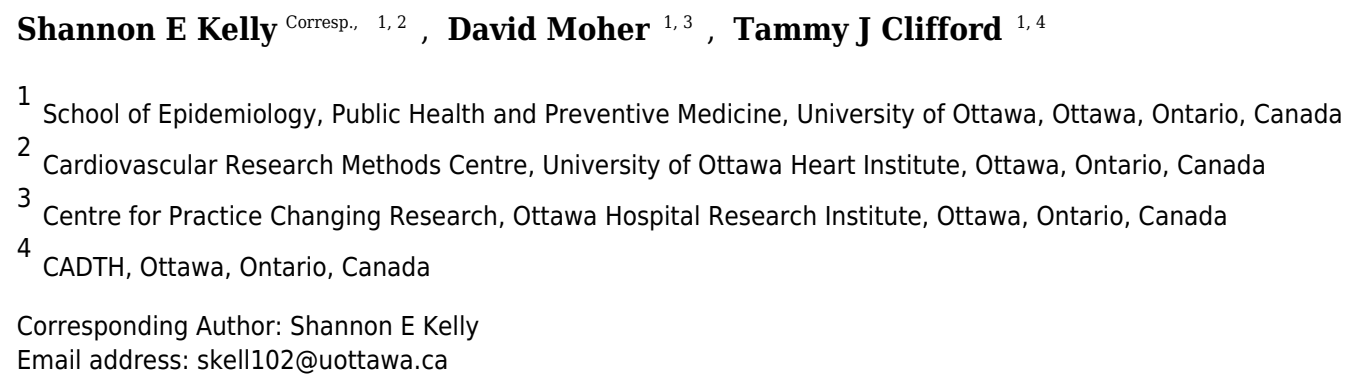

Background: Rapid reviews expedite the knowledge synthesis process with the goal of providing timely information to healthcare decision-makers who want to use evidence-informed policy and practice approaches. A range of opinions viewpoints on rapid reviews is thought to exist; however, no research to date has formally captured these views. This paper aims to explore evidence producer and knowledge user attitudes and perceptions towards rapid reviews.

Methods: A Q methodology study was conducted to identify central viewpoints about rapid reviews based on a broad topic discourse. Participants rank-ordered 50 text statements and explained their Qsort in free-text comments. Individual Q-sorts were analysed using Q-Assessor (statistical method: factor analysis with varimax rotation). Factors, or salient viewpoints on rapid reviews, were identified, interpreted and described.

Results: Analysis of the 11 individual Q sorts identified 3 prominent viewpoints: Factor A cautions against the use of study design labels to make judgements. Factor B maintains that rapid reviews should be the exception and not the rule. Factor $C$ focuses on the practical needs of the end-user over the review process.

Conclusion: Results show that there are opposing viewpoints on rapid reviews, yet some unity exists. The three factors described offer insight into how and why various stakeholders act as they do and what issues may need to be resolved before increase uptake of the evidence from rapid reviews can be realized in healthcare decision-making environments. 


\section{Expediting evidence synthesis for healthcare decision-making: Exploring attitudes and perceptions towards rapid reviews using Q methodology}

Shannon E Kelly ${ }^{1,2}$, David Moher ${ }^{1,3}$, Tammy J Clifford ${ }^{1,4}$

${ }^{1}$ School of Epidemiology, Public Health and Preventive Medicine, University of Ottawa, Ottawa, Ontario, Canada

${ }^{2}$ Cardiovascular Research Methods Centre, University of Ottawa Heart Institute, Ottawa, Ontario, Canada

${ }^{3}$ Centre for Practice Changing Research, Ottawa Hospital Research Institute, Ottawa, Ontario, Canada

${ }^{4}$ CADTH, Ottawa, Ontario, Canada

Corresponding Author:

Shannon Kelly ${ }^{1,2}$

H2267A - 40 Ruskin Street, Ottawa, Ontario, K1Y 4W7, Canada

Email address: skelly@ottawaheart.ca 


\section{INTRODUCTION}

2 The requirement for timely input to policy and healthcare decision-making encouraged evidence

3 producers to accelerate their processes, resulting in the approach often referred to as a rapid

4 review. Rapid reviews expedite the evidence synthesis process by streamlining or tailoring the

5 rigourous and explicit methods of a systematic review, and a variety of approaches may be

6 employed (1-10). Use of rapid reviews is seemingly increasing as organizations struggle to meet

7 the needs of knowledge users requesting timely evidence-informed decision support. Research

8 on rapid reviews is consequently expanding in parallel as investigators endeavour to fill

9 documented knowledge gaps by attempting to define and validate approaches and quantify the implications and impact of use $(1,11)$. Despite these efforts, little is known about the opinions or

11 views of both evidence producers and knowledge users towards rapid reviews.

12 Although informal opinions about rapid review approaches seem to abound, no formal study of these views exists in the literature. Early on, as this type of approach was new to the evidence synthesis landscape, there appeared to be a certain stigma attached to rapid reviews. The general perception at that time was that a rapid review was a "quick and dirty" systematic review, and there was pushback from conservatives in the research methodology community despite uptake from knowledge users. Previous work has highlighted common themes associated with rapid reviews: the aim to inform health care decision-makers (also called knowledge users), a deficit in reporting and transparency of conduct, and an unclear, heterogeneous of approaches that all fall under the broad umbrella term 'rapid review' (12-15). Yet, despite some central shortcomings, rapid response programs continue to thrive and expand internationally $(4,7)$. Although negative views may persist, it seems clear that there is a distinct group of supporters who continue to cautiously champion and encourage the use of rapid reviews to support evidence-informed 
24 decision making. No research to-date has attempted to capture these views, and we know very

25 little empirically how producers subjectively feel about rapid reviews and the risk of flawed

26

27

28

conclusions or 'less than best' data that may result from methodological concessions made while accelerating the evidence synthesis process. Similarly, no organized study to-date has measured the end-users opinions on rapid reviews, how they value this form of evidence, or their experience with rapid reviews in practice.

Though difficult to delineate, improved understanding of the prevailing or varying values, beliefs and attitudes pertaining to rapid reviews is essential, as mindset can influence action, conduct and uptake. Investigating attitude and perception is important for a few reasons. Cross (2005) states that attitude and opinion help to form cognitive relationships, which in turn may influence actions or conduct (16). In fact, attitude can be defined by a predisposition to act in a particular way (17). As such, positive thought may lead a producer or user to approach or value rapid reviews in a positive manner or predispose them to behave in a supportive fashion. Likewise, more cautious, or even pessimistic notions around rapid reviews may influence conduct, curb use, or sully their value which may limit impact. Comparing and contrasting these factors among and across the various stakeholder groups may serve to identify correlations in thought, similarities in experience or gaps in needs or methods. In addition, a comprehensive understanding of beliefs towards rapid reviews will further inform both evidence producers and researchers on how to continue to support the needs of decision-makers.

\section{A broader view of rapid reviews}

This study is part of a broader research program on rapid reviews which involved 3 independent, yet related studies, including: 1) a scoping review of rapid review samples to map characteristics and methods of rapid reviews and to check their adherence to conduct and reporting guidelines 
47 (18); 2) a modified-Delphi study aiming to identify key defining characteristics of rapid reviews

48 (Kelly 2016, Unpublished results); 3) the Q methodology study on attitudes and perceptions

49 reported here. The course of action from the associated study program contributed to the sharing

50 of knowledge and provided a gateway for an expanded discourse on rapid reviews; however,

51 opening the dialogue and merely summarizing the collective thought is insufficient. Hartling et

52 al. (2015) note the importance of studying end-user perspectives in their recent work (4). In order

53 to further our global understanding of rapid reviews, it is also important to study the attitudes and

54 traits of those who produce them. Given the absence of evidence from either perspective, a more

55 formal review is merited.

\section{Q methodology}

57 Fundamentally, Q methodology is a research methodology that allows for the systematic study of subjectivity (19). The method employs both qualitative and quantitative methods to reveal and detail viewpoints, values, attitudes, and opinions among a group of participants on a particular topic (20). A few key objectives underlie the use of a Q methodology; First, the goal of finding the range of communicated ideas on a topic, followed by exploration of the prevailing variations in it, and finally, to logically connect these variations in an orderly way to each other. Following individual rank-ordering of statements, connecting of viewpoints is completed statistically through an inversion of conventional factor analysis (20). The difference lies in the assessment of correlation of individuals rather than tests or mathematical variables. Correlation is done across viewpoints and ultimately mapped to results labelled 'factors'. Following a careful and methodological interpretation, the resulting factors represent the participants' subjectivity on the topic, and tell a specific 'story' about their beliefs, values and perceptions (Figure 1) (19). 
Figure 1. Steps in Q Methodology (Adapted from Amin, 2000 (16)).

71 There are a small number of fundamental steps essential to the Q methodology(20). First,

collection of a broad, balanced sample of statements, referred to as the 'concourse', which represents all relevant dialogue about a topic of interest. The concourse is then further refined into a set of statements called the 'Q set'. The Q set broadly represents the opinion field for the topic described in the concourse and is balanced to ensure that individual items capture each idea without gaps or unnecessary overlap. It is important to note that balance in this sense does not mean that half of the statements are positive and the other half negative.

There is no solitary or exact way to produce a Q set. According to theory, it must be "tailored to the requirements of the study and the demands of the research question it is seeking to answer" (21). Development of the Q set can generally be done in a structured or unstructured manner. In a structured Q set, relevant subject matter is organized into themes or ideas based on research or observation or possibly, a preconceived theory. Items in the Q set are then generated to ensure that all relevant themes identified are covered. An unstructured Q set is also constructed based on the entirety of relevant themes and key ideas collected. The more flexible sampling process allows for arrangement of the Q set into a series of statements representative of the whole population in the concourse. Arguably there is more freedom with this method of Q set definition; however, efforts must be made to maintain a rigourous and comprehensive process. All Q sets are ultimately judged on comprehensiveness, representativeness and balance in relation to the research question while remaining unbiased to any particular viewpoint (3).

Next, a set of participants is selected who are referred to as the P set. Q methodology allows for use of small sample size as the aim is not to estimate population statistics, but rather to maximize 
92 diverse viewpoints and show the existence of these views (19). Q-sets of between 40 and 80

93 items have become standard as anything less may not represent the views or be too limiting (22,

$9423)$.

95 Participants are asked to review the Q set of randomly ordered statements and to rank each from

96 those they agree with the most to those they disagree with the most. This sorting is done using a

97 pre-defined grid based on a quasi-normal distribution. This is a standard means of simplifying

98 the following statistical procedure without biasing the factors that are interpreted (24). Both

99 online and mail-in approaches have been validated against traditional in-person interviews and

100 sorting. Studies have shown reliability and validity, with no difference in outcomes associated

101 with changing the method of administration (25). Online methods also allow for access to a

102 theoretically relevant sample of individuals that have a much broader national or international

103 distribution. Although Q-methodology gives the impression that it is a difficult methodology for

104 a lay-person to self-administer, studies have shown that parents, clinicians and the general public

105 respond well when instructions are clear $(21,25)$. This method is referred to as ' $\mathrm{Q}$ sorting'.

106 Following the Q sort, the data are analysed using factor analysis. Finally, factor interpretation is

107 carried out based on the results of the analysis phase.

108 The main goal of this study is to gauge how producers and knowledge-users feel about rapid

109 reviews, and explore the range of opinion on this useful evidence synthesis approach. In

110 addition, this study hopes to gain an understanding of whether these types of evidence

111 summaries are valued, appropriate, or if certain misgivings persist given the lack of validation

112 against gold standard methods. This paper presents the results of an explorative Q methodology

113 study of evidence producer and knowledge user attitudes and perceptions towards rapid reviews. 


\section{MATERIALS AND METHODS}

115 Q methodology was used to identify participants' collective attitudes and perceptions towards 116 rapid reviews.

\section{Setting}

118 Web-based Q sorting using Q-Assessor software with e-mail communication to participants (26).

\section{Defining the $\mathbf{Q}$ set}

120 A broad, comprehensive concourse representing a range of views towards rapid review was 121 derived from a wide-range of sources prior to the refinement of the $\mathrm{Q}$ set $(1,3,5,27-30)$. The 122 goal at this stage was to document as many viewpoints as possible pertaining to rapid reviews, 123 before refining these views into the list of opinion statements that form the Q set. Following this 124 approach, statements for the concourse were gathered or extracted using 1) a small set of 125 academic papers focused on rapid reviews reviewed in detail for author viewpoints; 2) conference proceedings where opinions and beliefs towards rapid reviews were transcribed in-

127 person from plenary, oral or panel presentations, and posters or abstracts were examined for 128 relevant opinions (questions or comments related to expediting evidence production during these 129 proceedings were also transcribed); 3) detailed review of online resources, social media (Twitter) 130 and electronic mailing lists and, 4) in-person consultation in the form of unstructured dialogue 131 with experts in evidence synthesis, including both users and producers of rapid reviews. The first 132 author attended the 2011 to 2014 Canadian Agency for Drugs and Technologies and Health 133 (CADTH) Symposiums, the 2013 International Cochrane Colloquium in Quebec City, Quebec, 134 and the 2014 Cochrane Canada Symposium in Ottawa, Ontario in order to document and record concourse statements. 
136 A semi-structured approach was used to reduce the total number of viewpoints in the concourse

137 to a balanced and representative Q set, eliminating repeated thoughts and redundancies. Items, or

138 individual opinion statements, were organized based on similar themes and viewpoints pertaining

139 to rapid reviews, including: timing, scope, perceived or actual bias resulting from tailoring

140 methods, transparency or reporting, inconsistency in approaches used to expedite evidence

141 synthesis, ways of tailoring methods, breadth or depth of evidence collected, utility of rapid

142 reviews in decision-making, appropriateness, validity of results, confidence in results, and

143 suggestions for future research in this area. Best practices for evidence synthesis (the gold

144 standard for synthesizing evidence) were also factored in to the Q-set statements from the

145 concourse (rigour, precision of estimate, reproducibility, transparency, explicit methods). Views

146 in the concourse from both producers and knowledge users also reflected general support for the

147 use of rapid reviews as well as hesitance or reserved judgement until further research is

148 published. Statements from the concourse were refined based on these themes and viewpoints

149 into a Q set of 50 items. This process was carried out by the first author in consultation with the

150 second and third authors.

151 For the purposes of this study, the following terminology was used: 1) Evidence producers are

152 anyone who carries out research activities and may be involved in evidence synthesis activities in

153 any capacity; 2) Knowledge users are those who are likely to use the information generated

154 through research to make informed decisions about health policies, programs, and/or practices;

155 3) Systematic reviews employ rigorous methods, such as those prescribed by the Cochrane

156 Collaboration, to summarize research evidence; 4) Rapid reviews generally follow similar

157 systematic methods, but these methods are tailored or modified in order to synthesize research

158 evidence in a more timely manner to meet the particular needs of knowledge users; and, 5) 
159 Health technology assessment is a broader, policy-based assessment of medical, social, ethical 160 and economic implications of development, diffusion and use of health technologies.

\section{Assembling the P set}

162 In order to maximize the possibility that a variety of perspectives could be articulated, and to 163 ensure that both knowledge users and producers were captured in the sample, participants in this 164 study were identified using a purposeful sampling approach. Study authors used a publicly 165 available list of attendees present at four consecutive years of the annual CADTH symposium to 166 identify both producers and knowledge users. The goal was to capture participants actively

167 involved in the areas of policy making, program decision-making, healthcare delivery and 168 research. Ineligible attendees were removed, including those who were industry representatives, 169 administrative staff, students, patient group association representatives and those who attended 170 the annual symposium only once in the four years reviewed. The initial recruitment goal was set 171 at 50 participants.

172 Representatives were then split into two distinct lists representing knowledge users and 173 producers and randomly ordered. Starting with the first person in each ordered list, those without 174 a publicly accessible email address were removed in order to be consistent with Canada's anti175 spam legislation that came into effect July 1, 2014 (http://fightspam.gc.ca). A large proportion of 176 CADTH research staff were included in the sample. As such, the proportion of researchers was 177 restricted to no more than $20 \%$ CADTH staff in order to maintain an unbiased grouping. After 178 obtaining approval from the Ottawa Hospital Research Ethics Board (protocol \#20120143-01H), 179 the first 25 individuals in each list were invited to participate using standardized email invitations 180 containing a letter outlining the study objectives, methods, expected time commitment, consent 
181 information, and a link to the online Q sort. Replacement invitations were sent to new potential

182 participants following a refusal or when no response was received from an invitee after 2

183 reminders sent at 7 day intervals.

184 The final P set consisted of 11 participants (53 invitations, response rate 20.8\%). Five invitations

185 were declined for unspecified reasons, 4 email invitations bounced and 2 potential participants

186 contacted the first author to decline for personal reasons, including lack of time $(\mathrm{n}=1)$, lack of

187 knowledge on rapid reviews $(\mathrm{n}=1)$. A profile of the expert panel is provided in Table 1.

188 Participants were predominantly female (72.7\%) with doctoral degrees $(63.6 \%)$ and aged 35 or

189 older (81.8\%). All were from Canada. Two knowledge users, 8 producers and 1 respondent who

190 did not consider themselves part of either category responded. Names of participants have not

191 been identified in order to maintain the anonymity agreed upon as a condition of participation.

192 Participants were not compensated.

Table 1. Profile of the producers and knowledge users $(n=11)$.

194 Although the sample size may be considered small for a conventional factor analysis, the

195 fundamental ideology and statistical technique underlying the Q method maintain that as long as

196 a potential range of views are covered, small sample sizes may be adequate for the level of

197 understanding sought out (20).

\section{Q sort table}

199 A predetermined grid, or Q sort table, based on a quasi-normal distribution was used (Figure 2).

200 The table consisted of 50 cells spread across 7 columns of varying length matching the number

201 of statements in the Q set. Scores of +3 (most agree) and -3 (most disagree) were assigned to 
202 anchor the extreme limits of the Q sort table, with sequential label numbering heading each

203 column, including a value of ' 0 ' for neutral.

204

205

206

207

208

209

210

211

212

213

214

215

216

217

218

219

220

221

222

223

224

225

Figure 1. Fixed distribution for the Q-set

Five individuals piloted the instructions, Q set and Q sort online using Q-Assessor, a web-based software dedicated to Q methodology that was customized for this study (26). Instructions were examined for clarity, feasibility, and to ensure that the practical elements of design were appropriate. Q set was examined by the same individuals and the study team for content validity. Feedback did not result in a reduction or increase in the number of statements, only minor adjustments to wording for clarity or ambiguity.

\section{$Q$ sort process}

Participants completed the online Q sorting process using Q-Assessor (26). On the initial page, they were provided with an overview of study terminology, reminded of the study objectives, and asked to proceed to a preliminary Q sort of the 50 statements on rapid reviews. Next, participants sorted the statements into broad categories of "agree", "disagree" and "neutral" based on their own understanding, opinions and perceptions of rapid reviews. In the subsequent step, the Q sort table was presented (Figure 2). Participants were first asked to consider the range of opinion represented in the Q set while selecting and placing the statements they most strongly agree $(n=2)$ or disagree $(n=2)$ with onto the extremes of the table. After that, they free-sorted the remaining 46 statements, moving and reviewing the position of each until satisfied that they best reflected their own views. Finally, participants were asked to qualify selection of the four strongest views ranked in their Q sort by providing a succinct explanation for their choices in a free-text comment box. A brief profile questionnaire was also administered. Based on pilot 
226 testing, it was estimated that the time to completion would be between 20 and 25 minutes. Actual

227 time to completion was 45 minutes, on average.

\section{Analysis and factor interpretation}

229 Q sorts entered by participants were analyzed using by-person factor analysis (i.e., statistical analysis is based on the individual, instead of a statement, characteristic or trait) in Q-Assessor. Initially, a correlation matrix was created to identify patterns of agreement and disagreement

232 across the individual Q sorts. Correlations larger than 1.96 times the standard error $(1 / \sqrt{n}$, where $233 \mathrm{n}$ is the number of statements) were used to identify significant relationships in the data (19).

234 Factor extraction was initiated by calculating centroid factor loadings from the data. Positive and 235 negative associations between each Q sort and the seven preliminary factors were explored.

236 Eigenvalues, variance (total and by factor) and communality $\left(\mathrm{h}^{2}\right)$ were also calculated.

237 Eigenvalues and individual variance represent the strength of the factor extracted and its

238 potential explanatory power, with a higher value representing superior factor choices (20).

239 Communality is a measure of how much an individual Q sort holds in common with the other

240 sorts in the study and is used for comparing and contrasting individual response across the initial 241 (unrotated) factors.

242 Following a careful assessment of the preliminary factor loadings across a number of potential 243 factor arrangements and their associated statistics, primary factors were extracted. In order to be 244 considered, factors had to have an eigenvalue $\geq 1.00$ and at least one significant loading as 245 assessed by the Fuerntratt criterion following a varimax rotation of latent factors (31).

246 Additionally, the group of residual factors had to account for at least $40 \%$ of the total variance in 247 the Q sorts (32). 
248 Prior to factor interpretation, factor arrays (Table 2) and normalized weighted average statement

249 scores (z-score), or factor scores, were calculated. Statements with a significant factor score

$250(\mathrm{p}<0.05)$ were considered distinguishing for a factor (25). Factors were then qualitatively

251 interpreted based on the systematic and methodical approach to factor interpretation using the

252 organization system described by Watt et al. (Chapter 7) (20).

Table 2. Q-set statements and factor array

\section{RESULTS}

255 All 11 participants completed the Q sort process and answered the open-ended interview

256 questions following (completion rate 100\%). Three study factors were identified following a by-

257 person factor analysis of 11 Q sorts. Study factors were categorized as Factor A “Don't judge a

258 book by its cover", Factor B “Gold standard or bust" and Factor C “The pragmatist(s)".

259 Following varimax rotation, the three extracted factors explained $46.1 \%$ of the total variance in

260 this study. All 11 Q sorts loaded significantly on one of the three factors and none of the Q sorts

261 were confounded (meaning that none of the Q sorts loaded significantly on more than one

262 factor). All three factors had eigenvalues greater than 1.00, however, only two of the factors had

263 two or more significant Q sorts and can be considered exemplar individual factors. Table 3

264 shows the characteristics for the factors. The composite reliability coefficients $\left(\mathrm{r}_{\mathrm{c}}\right)$ indicated

265 construct validity for each factor as all values acceded the acceptable threshold of 0.70 (33).

267 Each factor, or salient perspective on rapid reviews, emerged from the attitudes and beliefs of the 268 participating producers and knowledge users. Factors were 'named' according to their defining 269 characteristics and following a careful, comprehensive interpretation of the factor arrays, scores and rankings. Participant profile information and results from the open-ended questions were 
271 also considered during interpretation. A description of each factor is presented with summary

272 details of the participants who loaded significantly on the factor. Rankings of relevant items are

273 provided. For example $(+3)$ indicates that a statement is ranked in the +3 position which

274 represents agreement in the factor array Q sort.

275 Factor A. “Don't judge a book by its cover"

276 Factor A explained $24.5 \%$ of the total study variance. Eight of 11 participants significantly

277 loaded on this factor. The majority of responses $(87.5 \%)$ were from evidence producers.

278 This group was characterized by their view that we need to look more in-depth at the value or

279 quality of individual review as opposed to a global assessment based on the labels traditionally

280 employed to distinguish between evidence synthesis products (i.e. systematic review, rapid

281 review). They had strong agreement $(+3)$ with two statements in particular: "All evidence

282 synthesis products, including rapid reviews, [systematic reviews, or health technology

283 assessments], can be conducted very well or very poorly" and "A well-conducted rapid review

284 may produce better evidence than a poorly conducted systematic review”. They similarly

285 disagreed (-2) with the statement that "a rapid review cannot be a systematic review". Quality

286 and value were again referenced in factor A $(+2)$ through participant agreement with the

287 statement "Rapid reviews and all other evidence synthesis products hold the same value as long

288 as they retain the core value of being transparent in conduct, include the highest quality evidence

289 available and present results with a qualification on the strength of evidence". Participants

290 defined by this factor may prescribe to a manifesto that acknowledges 'the good, the bad and the

291 ugly' in all types of evidence synthesis products. 
292 The relationship between time and quality were also common themes in factor A. Participants

293 agreed that value and quality were not tied to the length of time taken to complete a review, no

294 matter how long or short. They agreed $(+2)$ with the statement "A good quality review of

295 evidence is determined by the methods used, not by the speed at which it is completed" and

296 disagreed (-3) with the statement "The more time spent conducting the review of the evidence,

297 the more valid the results of the review will be".

298 This group agreed $(+2)$ that "Using rapid reviews to inform decisions is better than using no

299 evidence at all" but that minimum reporting standards are desirable (+2) (e.g. A PRISMA

300 statement for rapid reviews). This is supported by the disagreement (-2) documented for

301 "Reporting of the results of rapid reviews must be tailored to the knowledge user(s) who

302 commissioned the review".

303

304

305

306

307

308

309

310

311

312

\section{Factor B. "Gold standard or bust"}

Factor B explained $9.6 \%$ of the total study variance. Two participants significantly loaded on this factor, one producer and one knowledge user.

This group strongly believed in the gold standard systematic review to meet the needs of knowledge-users, and that use of rapid reviews should be the exception, and not the rule. They firmly hold the belief $(+3)$ that "deviating from accepted systematic review methods may introduce bias and impact the validity of the resulting rapid review, which may be an unacceptable risk for some for knowledge users" and that "rapid reviews cannot be systematic reviews". They also strongly disagreed (-3) that it is always appropriate to conduct rapid reviews. They agree $(+2)$ with conducting a comprehensive systematic review of all available evidence 
313 when time allows, and that rapid reviews do not replace systematic reviews or health technology

314 assessments. They were also clear in their disagreement (-2) with the statement "The opportunity

315 cost of a comprehensive systematic review or health technology assessment is too high and it is

316 more advantageous to conduct rapid reviews when timeliness is a factor" and generally agreed

$317(+1)$ that "Rapid reviews should only be conducted when the alternate option is the use of no

318 evidence to inform a decision". Participants also endorsed the view $(+1)$ that "Rapids reviews are

319 'quick and dirty' systematic reviews", which participants in Factors A and C both disagreed with

320 (-2). This sentiment is repeated in their disagreement (-2) with the principle suggested by "A

321 well-conducted rapid review may produce better evidence than a poorly conducted systematic

322 review"

323 Factor B, more than other groups, asserted that additional research in the area of rapid reviews is

324 warranted. They also disagreed with statements pertaining to standardization of rapid review

325 methods conflicting with the needs of knowledge users. They are neutral in their beliefs that

326 rapid reviews meet the needs of decision-makers, and strongly (-2) disagree with the idea that

327 'Knowledge users don't always need all of the evidence, they just need the best evidence to

328 support their decision, and what is 'best evidence' is specific to the knowledge user". To this

329 group, it appears that a systematic review should always be considered 'best'.

\section{Factor C. “The Pragmatist”}

331 Factor C explained $11.9 \%$ of the total study variance. One participant, an evidence producer, 332 significantly loaded on this factor. 
333 This factor was characterized by a focus on the pragmatic needs of the knowledge user, balanced

334 with the value of tailored rapid reviews and the inherent risk of bias that may accompany their

335 use in decision-making processes. In opposition to those in Factor B, the participant felt strongly

$336(+3)$ that "Knowledge users don't always need all of the evidence, they just need the best

337 evidence to support their decision, and what is 'best evidence' is specific to the knowledge user".

338 The evidence producer also strongly agreed $(+2)$ that rapid reviews meet the needs of knowledge

339 users and must be tailored to the individual specific needs of those commissioning the review

340 (+3). The single participant disagreed (-3) that "When time allows, a comprehensive systematic

341 review of all available evidence should always be conducted".

342 The Factor $\mathrm{C}$ viewpoint is also pragmatic in that the participant seemed to accept that use of

343 rapid review approach may bring with it some risk. There is an emphasis in this viewpoint $(+2)$

344 that "The value of rapid reviews in the context of emergent decision-making needs outweighs the

345 disadvantages or risk of bias and potentially 'imperfect' evidence" and this is balanced with the

346 requirement $(+2)$ that transparency of process is important as it allows the end user to make their

347 own assessment on validity and appropriateness. This is supported by the belief that there should

348 be minimum standards for the methodological conduct of rapid reviews but disagreement $(-2)$

349 that "It is appropriate to endeavor to define a single, unique methodology for rapid reviews". The

350 evidence producer also admits they believe it is difficult to tell a rapid review from a systematic

351 review unless it is explicitly stated $(+1)$. This may carry forward the idea that although this

352 individual likes to have the option of a pragmatic approach to meet their needs, they also require

353 as much information about the methods used so they know how much confidence they can place

354 on the results. 
355 There was some overlap with the perspectives defined by Factor A. The participant firmly

356 disagreed (-2) that "A rapid review cannot be a systematic review" and that "Rapid reviews are

357 quick and dirty systematic reviews".

\section{Consensus and disagreement statements}

359 Participants equally agreed or disagreed on several statements that were not distinguishable

360 across factors, referred to as 'consensus statements' (Table 4). They generally agreed with the

361 statement "Rapid reviews mean different things to different people". There was broad

362 disagreement with three statements: "It is appropriate to endeavor to define a single, unique

363 methodology for rapid reviews", "Any review of evidence that takes longer than 3 months to

364 produce is not a rapid review." and "Any review of evidence that takes longer than 1 month to

365 produce is not a rapid review". All participants strongly disagreed with the statement "Rapid

366 reviews should only precede a more comprehensive and rigorous systematic review". There was

367 no concordance across factors when disagreement was considered; however, Factors A and C

368 tended to disagree with statements in a similar pattern with varying degrees of magnitude.

369

370

371

372

373

374

375

376

Table 4. Statements showing agreement and disagreement across factors

\section{DISCUSSION}

This research explored the attitudes and perceptions towards rapid reviews in a group of evidence producers and knowledge users. Analysis of the Q sorts identified 3 salient viewpoints which represent the broad spectrum of health care decision-makers and those who synthesize evidence to inform them. Factor A cautions against using labels to judge the quality or value or rapid reviews (or any other evidence synthesis products) and asserts that these variables should be assessed on an individual basis before appropriateness and worth can be gauged. Those 
377 prescribing to Factor B firmly hold the concepts of rigour and consistency of process found in a

378 comprehensive systematic review true, and maintains that rapid reviews should be the exception

379 and not the rule. Factor $\mathrm{C}$ has a focus on the pragmatic needs of the end-user instead of process,

380 and is content balancing any risk that may be introduced by tailoring of methods with the

381 imperative need for timely review of evidence. Importantly, results show that there are quite

382 opposing viewpoints on rapid reviews, but that some commonality across these perspectives also

383 exists. The three factors described here are not necessarily exhaustive of the attitudes and

384 perceptions held about rapid reviews; however, the relatively clear viewpoints may be valuable

385

386

387

388

389

390

391

392

393

394

395

396

397

398

399

on their own as they offer insight into how and why various stakeholders act as they do and what issues may need to be resolved to increase uptake of evidence from rapid reviews. To our knowledge this is the first study to specifically address this facet of rapid reviews.

Results of this study indicate that significant gaps still exist in perceived knowledge about rapid reviews. Most participants (Factor A, predominantly evidence producers) felt uncomfortable using the broad study labels to place any assessment of value or quality on the products falling under the nomenclature umbrella of 'rapid review'. Additionally, there was broad consensus across all of the factors extracted that the term 'rapid review' means different things to different people. The notion that all rapid reviews are not created equal is not a novel finding, but rather a commonly asserted trait consistent with previous reports $(1,3,5,28,34)$. Our results indicate that most participants, regardless of viewpoint are well-aware of the heterogeneous range of approaches used to conduct rapid reviews, and that there is no standard or accepted way to carry out an accelerated evidence synthesis. In truth, it is still unclear whether rapid reviews should aspire to any standard at all. Participants in this study, a small sample of mostly evidence producers, stipulated that it is inappropriate to consider a single methodology for rapid reviews, 
400 which indicates that future studies should focus on more acutely describing the range of

401 approaches captured by the term "rapid review" with the goal of providing some clarity to end-

402 users. This requirement for multiple approaches has been put in practice by health technology

403 assessment agencies like the Canadian Agency for Drugs and Technologies in Health (CADTH)

404 and Health Quality Ontario who have evolved their internal rapid response and review programs

405 into multi-product offerings. Recent endeavors by Moher (2015) and Hartling (2015) suggest

406 between 4 and 7 functional groupings of rapid review exist and this provides a good basis for

407 further study of the different approaches going forward $(4,13)$. It is still uncertain if the ability to

408 form an explicit definition for rapid reviews in hindered because of the varied approaches that

409 must be captured by it.

410 Research can "both communicate and miscommunicate" according to Glaziou et al. (2014), and

411 research that is not adequately reported is at risk of becoming what the authors refer to as

412 'research waste' of time and resources (35). Transparency is central to the creation and

413 evaluation of high quality research evidence, and something that rapid reviews are not well

414 known for (3). Providing sufficient information to end-users on research process and results is

415 arguably more important for rapid reviews given that they are designed specifically to inform

416 policy and practice and are based upon deviations from some accepted evidence synthesis

417 practices. Results from analysis of the viewpoints in factor A shows that there is a desire by

418 researchers to make their own judgements on the potential value and quality of rapid review

419 products; However, Ganaan (2010), Harker (2012) and most recently Hartling (2015) have

420 pointed out that inconsistencies in reporting and conduct make it difficult for knowledge users to

421 apply these judgements. The hesitancy of participants in factors A and B to fully endorse rapid

422 review methodology without caveat suggests that transparency is a pressing factor that must be 
423 addressed. This raises the question of whether extensions to current reporting guidelines (e.g.,

424 PRISMA, PRISMA-P) or conduct checklists (e.g., AMSTAR) are desired, or required to

425 encourage higher quality reporting. The opinions expressed in this study serve to remind

426 researchers, academic and non-profit organizations, HTA agencies, and editors of journals that

427 we need to do better when it comes to transparent reporting of research process and methods $428 \quad$ (36).

429 Knowledge users are faced with a challenge when it comes to rapid reviews. They must decide

430 whether it is acceptable to trade-off the timely receipt of evidence with the risk that

431 comprehensiveness of the end product may be compromised (10). We found that some

432 stakeholders are accepting of this perceived trade-off (Factors A and C) than others (Factor B)

433 when timely evidence sunthesis is required. Yet, there is little empirical evidence quantifying the

434 impact of the methodological shortcuts used to expedite the review process. $(1,2,5,7,29,30)$.

435 Following the completion of this study, Pham (2016) reported three agri-food case studies

436 investigating the impact of applying methodological shorts-cuts to expedite the systematic

437 review process. The shortcuts resulted in study omissions, a reduction in the number of cases

438 where where meta-analysis was possible and generally less-precise pooled effect estimates (37).

439 Despite this, Hartling and Polisena (2015) both point out that there are many well-established

440 rapid response programs internationally which underscores that the risk associated with short-

441 cuts is acceptable for certain end-users in specific situations $(4,7)$. There may be situations

442 where rapid reviews are inappropriate, as Coates and Polisena (2015) suggest, such as those

443 where there may be legal implications (7), or where evidence is required to feed into the

444 development of clinical practice guidelines (12). More research is warranted to clarify how 
445 decision-makers weigh these risks, to gauge when the risk of erroneous decision-making is too

446 high, and which situations in particular are inappropriate for rapid review.

447 Factors extracted also showed opposing opinions on whether a rapid review can, in fact, be a

448 systematic review. While it is clear that rapid reviews aspire to a standard, it is unclear what that 449 standard actually is. Methodologically, we know that it is sometimes feasible for systematic 450 processes to be sped up if resources are added. Moher (2015) provides a typology of rapid 451 reviews that includes a category for 'traditional' systematic reviews done quickly (13). Due to

452 poor reporting, we are often unable to tell whether a product like this has been tailored, or 453 whether additional project resources were added to meet an expedited timeline. Theoretically a 454 split exists amongst the various stakeholders too. Some fundamentally believe that if a product is 455 a rapid review, by very nature then, it cannot also be systematic - which is equivalent to saying a 456 rapid systematic review is an oxymoron, even though this term is often used for accelerated 457 syntheses (38). Based on the results of the study by Yuan et al., comparisons of rapid reviews to 458 'full', 'traditional' or 'gold standard' systematic reviews are common, and provide a notional 459 frame of reference on which we can judge rapid review conduct, reporting and outcomes (39).

460 We currently have little empirical evidence differentiating rapid reviews from systematic 461 reviews, and no prospective research to-date has quantified differences between the two review 462 types $(15,30)$. Simply put, at this time we do not know when a systematic review becomes 463 'unsystematic'. Further, we may be relating rapid reviews back to a frame of reference which 464 itself is flawed. Systematic reviews, with the possible exception of those carried out by the 465 Cochrane collaboration, have been noted to have extreme variations in conduct, quality and 466 reporting. Future research projects should aim to better quantify potential differences amongst 
467 these review types and to determine if assessing the quality and conduct of rapid reviews against

468 this benchmark is fair or appropriate.

469 Another unique view stemming from this study relates to concepts of quality and time.

470 Participants in Factor A specifically agreed that quality of a rapid review is not inherently tied to

471 the time taken to complete the work, in contrast to the view of those in Factor $\mathrm{C}$ who held the

472 opposite. Evidence actually supports the opinion of those in Factor C. Harker (2012), Hartling

473 and Kelly (2015) have all examined samples of rapid reviews in depth, evaluated their quality

474 and balanced this measure against the times taken to produce the reviews $(3,4,18)$. Results have

475 consistently shown a relationship between time and quality of reporting or conduct. Additional

476 research is needed to confirm these findings across the typologies of rapid review approaches

477 proposed by both Harker and Moher to investigate if time variables may be confounded by the 478 approach.

\section{Strengths and Limitations}

480 The strengths in this study lie inherently with the methodology. Q methodology was selected for 481 use in this project over other methods (e.g., simple questionnaire, interviews) because it offers

482 the means to study subjective topics in a more systematic and rigorous manner. No other method 483 explored enabled statements or qualitative descriptions to be quantified statistically using

484 validated research techniques. Q methodology also offers a cost-effective way to potentially

485 solicit opinion from a geographically diverse pool of evidence producers and knowledge users

486 within the confines of the research project timeline. 
487 It is important to acknowledge the potential limitations associated with this study. Although a

488 large sample size is not required for a Q methodological study, results are based on a small

489 number of participants who were predominantly evidence producers in Canada. No benefit was

490 derived from efforts aimed at improving response rates, including sampling with replacement,

491 use of user-friendly software accessible online or by portable devices (e.g., phone or tablet), and

492 weekly email reminders. Due to funding limitations, we were unable to continue sampling with

493 replacement until the desired study sample size of 50 was reached. The completion rate for those

494 who participated was $100 \%$ so we do not consider the time-to-completion of 45 minutes to be a

495 limiting factor; however, participant fatigue cannot be ruled out. The authors are aware of at least

496 one other formal study on rapid reviews being administered at the time of our study, and two

497 other informal email surveys on the topic were also circulated within 2 months of this study.

498 There would have been overlap in the evidence producers and users contacted, and this may have

499 contributed to our low response rate.

500 Effort was made during concourse development to collect representative viewpoints from both

501 users and producers of rapid reviews. Although the proportion of statements collected from each

502 group was not empirically measured, the views of evidence producers may have been more

503 common in the concourse or Q set. The number of evidence producers and knowledge users who

504 participated in this study was disproportionate. Only two knowledge users completed the study,

505 and their views and opinions are only expressed in a limited capacity in the Factors extracted.

506 For this reason, our results are more reflective of the evidence producers view and do not fully

507 capture knowledge users' perspectives or needs. 
508 The smaller than desired sample size meant that certain methodological concessions had to be

509 made when interpreting factors. Ideally, factors are defined by more than one Q sort, which we

510 did not achieve for Factor C. While the same result may have been achieved with a larger sample

511 size we cannot verify this claim with our study population. Although not intentional by design,

512 this study population allows for some insight into the thought processes of a small sample of

513 predominantly Canadian evidence producers. It is useful to keep in mind that evidence producers

514 who participated in this study were not geographically diverse, and provided rankings based on

515 their viewpoints tied to their own evidence synthesis products. Findings were interpreted by the

516 study authors who, while they have much experience with knowledge users and the

517 requirements of the healthcare decision-making processes, generally may identify more as

518 evidence producers which could have unintentionally influenced the way factors were interpreted

519 or results were presented.

520 Although we have identified a series of insightful viewpoints on rapid reviews, the range of

521 viewpoints is not globally reflective of views of the wider population of evidence producers and

522 stakeholders. Q methodology does not endeavour to make a claim of universal applicability or to

523 represent the views of a larger sample $(16,19)$. Q Methodology is also not intended to be a test

524 of difference, and accordingly, results for evidence producers and knowledge users could not be

525 compared and contrasted (4). While we cannot exclude the influence of this dynamic on our

526 results, historically, key memberships are not usually a defining influence on the generation of

527 factors. In order to truly identify the views of researchers and decision-makers independently,

528 two separate studies would need to be carried out using identical Q sets and procedures.

529 Additionally, the comments received post-Q-sort more or less represent a unique, 
530 complementary qualitative study. Outside of interpreting the factors, they offer a depth of

531 knowledge on this topic that deserves further exploration.

\section{CONCLUSIONS}

533 This study has shown that there are distinct subsets of evidence producers and users who value

534 and appreciate rapid reviews. At the same time, there are cautious segments of these populations

535 who acknowledge the place of rapid reviews in evidence-informed decision-making under

536 certain and exceptional circumstances. Much of the discourse in this study revolved around

537 central concepts of time and quality. While there is a growing body of evidence showing review

538 quality decreases with abbreviated timeframes, there are still key stakeholders who believe that

539 high-quality evidence can be synthesized in a timely manner. Empirical evaluation of the

540 methodological implications of applying a rapid review approach are currently restricted to small

541 case studies; therefore, a more fulsome study of these issues is necessary to explore whether

542 there is evidence to support the particular views and opinions expressed in this Q methodology

543 study.. Research may be required to better define our gold standard of reference for rapid

544 reviews before some of the uncertainties raised in dialogue by evidence producers and

545 knowledge users can be resolved. Further study of evidence producer and user attitudes and

546 opinions should be explored to evaluate whether the discourse changes as progress continues to

547 exemplify methods and practice.

\section{References}

549

550 
551

552

553

554

555

556

557

558

559

560

561

562

563

564

565

566

567

568

569

570

571

572

573

574

575

576

577

578

579

580

581

582

583

584

585

586

587

588

1. Ganann, R., Ciliska, D., Thomas, H. Expediting systematic reviews: methods and implications of rapid reviews. Implement Sci. 2010;5:56.

2. Hailey, D, Corabian, P, Harstall, C, Schneider, W. The use and impact of rapid health technology assessments. Int J Technol Assess Health Care. 2000;16:651 - 6.

3. Harker, J., Kleijnen, J. What is a rapid review? A methodological exploration of rapid reviews in Health Technology Assessments. Int J Evid Based Healthc. 2012 Dec;10(4):397-410.

4. Hartling, L., Guise, J. M., Kato, E., Anderson, J., Aronson, N., Belinson, S., Berliner, E., Dryden, D., Featherstone, R., Foisy, M., Mitchell, M., Motu'apuaka, M., Noorani, H., Paynter, R., Robinson, K. A., Schoelles, K., Umscheid, C. A., Whitlock, E. AHRQ Comparative Effectiveness Reviews. EPC Methods: An Exploration of Methods and Context for the Production of Rapid Reviews. Rockville (MD): Agency for Healthcare Research and Quality (US); 2015.

5. Khangura, S., Konnyu, K., Cushman, R., Grimshaw, J., Moher, D. Evidence summaries: the evolution of a rapid review approach. Syst Rev. 2012;1:10.

6. Khangura, S., Polisena, J., Clifford, T. J., Farrah, K., Kamel, C. Rapid review: an emerging approach to evidence synthesis in health technology assessment. Int J Technol Assess Health Care. 2014 Jan;30(1):20-7.

7. Polisena, J. Garritty, C. Kamel, C. Stevens, A. Abou-Setta, AM. Rapid review programs to support health care and policy decision making: a descriptive analysis of processes and methods. Syst Rev. [10.1186/s13643-015-0022-6]. 2015(4:26).

8. Tricco, A. C., Zarin, W., Antony, J., Hutton, B., Moher, D., Sherifali, D., Straus, S. E. An international survey and modified Delphi approach revealed numerous rapid review methods. J Clin Epidemiol. 2016 Feb;70:61-7.

9. Tricco, A. C., Antony, J., Zarin, W., Strifler, L., Ghassemi, M., Ivory, J., Perrier, L., Hutton, B., Moher, D., Straus, S. E. A scoping review of rapid review methods. BMC Med. 2015;13:224.

10. Featherstone, R. M., Dryden, D. M., Foisy, M., Guise, J. M., Mitchell, M. D., Paynter, R. A., Robinson, K. A., Umscheid, C. A., Hartling, L. Advancing knowledge of rapid reviews: an analysis of results, conclusions and recommendations from published review articles examining rapid reviews. Syst Rev. 2015;4(1):50.

11. Peterson, K., Floyd, N., Ferguson, L., Christensen, V., Helfand, M. User survey finds rapid evidence reviews increased uptake of evidence by Veterans Health Administration leadership to inform fastpaced health-system decision-making. Syst Rev. 2016;5(1):132.

12. Coates, V. Keynote Address: Rapid Reviews and Their Impact on Future Directions for Health Technology Assessment. . CADTH Rapid Reviews Summit, Vancouver, BC. [Internet]February 2015 .

13. Moher, D. Do we need a rapid review reporting guideline? Is PRISMA-P helpful when generating a RR protocol? Available at: https://www.cadth.ca/sites/default/files/events/David-Moher_PublishingRapid-Reviews Feb-4-2015.pdf. CADTH Rapid Reviews Summit, Vancouver, BC.

[Internet]February 2015. 
589

590

591

592

593

594

595

596

597

598

599

600

601

602

603

604

605

606

607

608

609

610

611

612

613

614

615

616

617

618

619

620

621

622

623

624

14. Schunemann, H. J., Hill, S. R., Kakad, M., Vist, G. E., Bellamy, R., Stockman, L., Wisloff, T. F., Del Mar, C., Hayden, F., Uyeki, T. M., Farrar, J., Yazdanpanah, Y., Zucker, H., Beigel, J., Chotpitayasunondh, T., Hien, T. T., Ozbay, B., Sugaya, N., Oxman, A. D. Transparent development of the WHO rapid advice guidelines. PLoS Med. 2007 May;4(5):e119.

15. Tricco, AC. Antony, J. Straus,SE. Systematic reviews vs. rapid reviews: What's the difference? Available at: https://www.cadth.ca/sites/default/files/events/Andrea-Tricco_RR-vs-SystematicReviews_Feb-4-2015.pdf. CADTH Rapid Reviews Summit, Vancouver, BC [Internet]February 2015 .

16. Cross, R. M. Exploring attitudes: the case for Q methodology. Health Educ Res. 2005 Apr;20(2):20613.

17. Ajzen, Icek, Fishbein, Martin. Attitudes and normative beliefs as factors influencing behavioral intentions. Journal of personality and social psychology. 1972;21(1):1.

18. Kelly, S. E., Moher, D., Clifford, T. J. Quality of conduct and reporting in rapid reviews: an exploration of compliance with PRISMA and AMSTAR guidelines. Syst Rev. 2016;5:79.

19. Brown, Steven R. A primer on Q methodology. Operant subjectivity. 1993;16(3/4):91-138.

20. Watts, Simon, Stenner, Paul. Doing Q methodological research: theory, method \& interpretation: Sage; 2012.

21. Akhtar-Danesh, N., Dehghan, M., Morrison, K. M., Fonseka, S. Parents' perceptions and attitudes on childhood obesity: a Q-methodology study. J Am Acad Nurse Pract. 2011 Feb;23(2):67-75.

22. Curt, Beryl C. Textuality and tectonics: Troubling social and psychological science: Open University Press; 1994.

23. Stainton Rogers, R., Stenner, Paul., Gleeson, Kate. Social Psychology: A Critical Agenda. Cambridge: Polity: Wiley; 1995.

24. Brown, Steven R. Political subjectivity: Applications of Q methodology in political science: Yale University Press; 1980.

25. Van Exel, Job, de Graaf, Gjalt. Q methodology: A sneak preview. Online document http://wwwqmethodologynet/PDF/Q-methodology. 2005.

26. Q-Assessor. [http://q-assessor.com].

27. Gough, D., Thomas, J., Oliver, S. Clarifying differences between review designs and methods. Syst Rev. 2012;1:28.

28. Hailey, D. A preliminary survey on the influence of rapid health technology assessments. Int J Technol Assess Health Care. 2009 Jul;25(3):415-8.

29. Watt, A., Cameron, A., Sturm, L., Lathlean, T., Babidge, W., Blamey, S., Facey, K., Hailey, D., Norderhaug, I., Maddern, G. Rapid reviews versus full systematic reviews: an inventory of current methods and practice in health technology assessment. Int J Technol Assess Health Care. 2008 Spring;24(2):133-9. 
625

626

627

628

629

630

631

632

633

634

635

636

637

638

639

640

641

642

643

644

645

646

647

648

649

650
30. Watt, A., Cameron, A., Sturm, L., Lathlean, T., Babidge, W., Blamey, S., Facey, K., Hailey, D., Norderhaug, I., Maddern, G. Rapid versus full systematic reviews: validity in clinical practice? ANZ J Surg. 2008 Nov;78(11):1037-40.

31. Fuerntratt, E. Zur Bestimmung der Anzahl interpretierbarer gemeinsamer Faktoren in Faktorenanalysen psychologischer Daten (The determination of the number of interpretable common factors in factor analysis of psychological data). Diagnostica. 1969;15:62-75.

32. Kline, Paul. An easy guide to factor analysis: Routledge; 2014.

33. Hair, JF, Andreson, RE, Tatham, RL, Black, WC. Multivariate data analysis. 5th (ed) Prentice-Hall Inc. Unites States of America. 1998.

34. Merlin, Tracy, Tamblyn, David, Ellery, Benjamin. What's in a name? Developing definitons for common health technology assessment product types of the International Network of Agencies for Health technology Assessment (INAHTA). International Journal of Technology Assessment in Health Care. 2014;30(04):430-7.

35. Glasziou, P., Altman, D. G., Bossuyt, P., Boutron, I., Clarke, M., Julious, S., Michie, S., Moher, D., Wager, E. Reducing waste from incomplete or unusable reports of biomedical research. Lancet. 2014 Jan 18;383(9913):267-76.

36. Shamseer, L., Stevens, A., Skidmore, B., Turner, L., Altman, D. G., Hirst, A., Hoey, J., Palepu, A., Simera, I., Schulz, K., Moher, D. Does journal endorsement of reporting guidelines influence the completeness of reporting of health research? A systematic review protocol. Syst Rev. 2012;1:24.

37. Pham, M. T., Waddell, L., Rajic, A., Sargeant, J. M., Papadopoulos, A., McEwen, S. A. Implications of applying methodological shortcuts to expedite systematic reviews: three case studies using systematic reviews from agri-food public health. Res Synth Methods. 2016 Jun 10.

38. Schunemann, H. J., Moja, L. Reviews: Rapid! Rapid! Rapid! ...and systematic. Syst Rev. 2015;4:4.

39. Yuan, Y., Hunt, R. H. Systematic reviews: the good, the bad, and the ugly. Am J Gastroenterol. 2009 May;104(5):1086-92. 
Table $\mathbf{1}$ (on next page)

Profile of the producers and knowledge users ( $n=11)$. 


\begin{tabular}{lc}
\hline $\begin{array}{l}\text { Geographic Location } \\
\text { Canada }\end{array}$ & $\mathbf{n}(\%)$ \\
Age: & $11(100)$ \\
18 to 35 & $\mathbf{n}(\%)$ \\
36 to 50 & $2(18.2)$ \\
50 or above & $4(36.4)$ \\
Sex: & $5(45.5)$ \\
\hline Female & $\mathbf{n}(\%)$ \\
Male & $8(72.7)$ \\
\hline Education: & $3(27.3)$ \\
Doctorate & $\mathbf{n}(\%)$ \\
Masters & $7(63.6)$ \\
Undergraduate & $3(27.3)$ \\
\hline Consider Themselves: & $1(9.1)$ \\
$\quad$ Researcher/Producer & $\mathbf{n}(\%)$ \\
Knowledge User & $8(72.7)$ \\
Neither & $2(18.2)$ \\
\hline Have ever been the author of & $1(9.1)$ \\
a rapid review & $\mathbf{n}(\%)$ \\
Yes & $7(63.6)$ \\
No & $4(36.4)$ \\
\hline Have used a rapid review to & \\
aid in a policy or decision- & $\mathbf{n}(\%)$ \\
making? & \\
Yes & $7(63.6)$ \\
No & $3(27.3)$ \\
Unsure & $1(0.9)$ \\
\hline
\end{tabular}

1 
Table 2 (on next page)

Q-set statements and factor array 


\begin{tabular}{|c|c|c|c|c|}
\hline \multirow[t]{2}{*}{ No. } & \multirow[t]{2}{*}{ Statements } & \multicolumn{3}{|c|}{ Factors } \\
\hline & & $\mathbf{A}$ & B & $\mathbf{C}$ \\
\hline 1 & The evidence from rapid reviews is good enough to inform low-risk, emergent policy or decision-making needs when the alternative is the use of no evidence. & 1 & 1 & 0 \\
\hline 2 & When time allows, a comprehensive systematic review of all available evidence should always be conducted. & -1 & 2 & -3 \\
\hline 3 & $\begin{array}{l}\text { Deviating from accepted systematic review methods may introduce bias and impact the validity of the resulting rapid review, which may be an unacceptable risk for some for knowledge } \\
\text { users. }\end{array}$ & 0 & 3 & 1 \\
\hline 4 & Further research comparing the methods and results of rapid reviews and systematic reviews is required before I decide how I feel about rapid reviews. & -1 & 2 & 0 \\
\hline 5 & Rapid reviews are too focused in scope and/or context to be generalizable to a variety of knowledge users. & -2 & -2 & -1 \\
\hline 6 & Rapid reviews mean different things to different people. & 1 & 2 & 1 \\
\hline 7 & Rapid reviews should only precede a more comprehensive and rigorous systematic review. & -3 & -3 & -3 \\
\hline 8 & The opportunity cost of a comprehensive SR or HTA is too high and it is more advantageous to conduct rapid reviews when timeliness is a factor. & 0 & -2 & 1 \\
\hline 9 & Rapid reviews do not replace SRs or HTAs. & 0 & 2 & 0 \\
\hline 10 & All evidence synthesis products, including rapid reviews, SRs, or HTAs, can be conducted very well or very poorly. & 3 & 2 & 0 \\
\hline 11 & Rapid reviews are comparable to SRs except they are done in a more timely fashion. & -1 & 1 & 1 \\
\hline 12 & Rapid reviews are 'quick and dirty' systematic reviews. & -2 & 1 & -2 \\
\hline 13 & Rapid reviews need to be tailored to the specific needs of the knowledge user. & 0 & 2 & 3 \\
\hline 14 & Rapid reviews meet the needs of knowledge users. & 1 & 0 & 2 \\
\hline 15 & There is a paucity of evidence on rapid reviews, so I cannot support or oppose their use in decision-making. & -1 & 0 & -1 \\
\hline 16 & There is so much overlap across the various evidence synthesis methods that I cannot generalize my opinion to favor one over the other without the context of the decision at hand. & 0 & 0 & -1 \\
\hline 17 & There is a risk involved in tailoring accepted SR methods to produce rapid reviews that we do not yet understand. & -1 & 0 & 2 \\
\hline 18 & Using rapid reviews to inform decisions is better than using no evidence at all. & 2 & 0 & 0 \\
\hline 19 & It is always appropriate to conduct a rapid review. & -2 & -3 & -1 \\
\hline 20 & $\begin{array}{l}\text { Rapid reviews and all other evidence synthesis products hold the same value as long as they retain the core value of being transparent in conduct, include the highest quality evidence } \\
\text { available and present results with a qualification on the strength of evidence. }\end{array}$ & 2 & -1 & 0 \\
\hline 21 & Appropriateness of a rapid review varies with the type of decision being made, and any financial, legal or other important contextual facets tied to the decision. & 2 & 1 & 1 \\
\hline 22 & My confidence in a rapid review is impacted by which methods are tailored to speed up the review process. & 1 & 0 & 0 \\
\hline 23 & My confidence in a rapid review is directly tied to results being presented and contextualized by the strength and applicability of the evidence. & 0 & 0 & -1 \\
\hline 24 & It is important to have minimum standards for the methodological conduct of rapid reviews. & 0 & 1 & 2 \\
\hline 25 & It is important to have minimum standards for the reporting of rapid reviews (e.g., a PRISMA-RR). & 2 & 0 & 0 \\
\hline 26 & Standardization of rapid review methods may conflict with the needs of knowledge users & 0 & -2 & 1 \\
\hline 27 & The value of rapid reviews in the context of emergent decision-making needs outweighs the disadvantages or risk of bias and potentially 'imperfect' evidence. & 1 & -1 & 2 \\
\hline 28 & Knowledge users don't always need all of the evidence, they just need the best evidence to support their decision, and what is 'best evidence' is specific to the knowledge user. & 1 & -2 & 3 \\
\hline
\end{tabular}




\begin{tabular}{|c|c|c|c|c|}
\hline \multirow[t]{2}{*}{ No. } & \multirow[t]{2}{*}{ Statements } & \multicolumn{3}{|c|}{ Factors } \\
\hline & & $\mathbf{A}$ & B & $\mathbf{C}$ \\
\hline 29 & Knowledge users do not fully understand the implications of streamlining evidence synthesis methods to produce a more timely evidence product. & 1 & 0 & 0 \\
\hline 30 & Reporting of the results of rapid reviews must be tailored to the knowledge user(s) who commissioned the review. & 0 & 0 & 0 \\
\hline 31 & Rapid reviews that omit an assessment of the quality of included studies are useless to knowledge users. & 0 & -1 & -1 \\
\hline 32 & Rapid reviews can be timely and valid, even when methodological concessions are made. & 1 & 1 & 1 \\
\hline 33 & $\begin{array}{l}\text { Transparency of process is more important than the actual methods used to produce rapid reviews, as transparency allows the end user to make their own assessment on validity and } \\
\text { appropriateness. }\end{array}$ & 0 & -2 & 2 \\
\hline 34 & It is appropriate to endeavor to define a single, unique methodology for rapid reviews. & -1 & -1 & -2 \\
\hline 35 & Rapid reviews are not a unique methodology, they are simply a variation of a systematic review that can fall anywhere on the continuum of evidence synthesis methods. & 1 & -1 & 0 \\
\hline 36 & $\begin{array}{l}\text { The results from a systematic review may not differ from those of a rapid review, but more research is needed to support this theory and quantify why results may be the same or } \\
\text { different. }\end{array}$ & 1 & 1 & 0 \\
\hline 37 & I put more confidence in evidence produced in a systematic review than of a rapid review. & -1 & 1 & -1 \\
\hline 38 & The more time spent conducting the review of the evidence, the more valid the results of the review will be. & -3 & -1 & -2 \\
\hline 39 & Achieving a precise estimate of effect (from a SR) may not inform the decision-at-hand any better than a general estimate of effect (produced by a rapid review). & 2 & -1 & 1 \\
\hline 40 & Rapid reviews should only be conducted when the alternate option is the use of no evidence to inform a decision. & -2 & 1 & -1 \\
\hline 41 & A well-conducted rapid review may produce better evidence than a poorly conducted systematic review. & 3 & -1 & 2 \\
\hline 42 & Any review of evidence that takes longer than 3 months to produce is not a rapid review. & -1 & -2 & -1 \\
\hline 43 & Any review of evidence that takes longer than 1 month to produce is not a rapid review. & -2 & -1 & -2 \\
\hline 44 & A rapid review must be justified with a valid rationale for both speeding up the process and tailoring rigourous methods for evidence synthesis. & -1 & 0 & 0 \\
\hline 45 & A good quality review of evidence is determined by the methods used, not by the speed at which it is completed. & 2 & 1 & 0 \\
\hline 46 & It is difficult to tell a rapid review from a systematic review unless very specific nomenclature is used in the title or description of methods. & -1 & 0 & 1 \\
\hline 47 & A rapid review cannot be a systematic review. & -2 & 3 & -2 \\
\hline 48 & 'Rapid review' is too broad a phrase - doing a review in a more timely way can only be relative to how long it takes the same team to produce a full systematic review. & 0 & -1 & -1 \\
\hline 49 & Producers are more concerned with the methodology and validity of rapid reviews than knowledge users. & 0 & 0 & 1 \\
\hline 50 & It is difficult to judge the validity of a rapid review as the reporting is often truncated and protocols are not published. & 0 & 0 & -2 \\
\hline
\end{tabular}


Table 3 (on next page)

Q Factor characteristics 


\begin{tabular}{lccc}
\hline Characteristics & \multicolumn{3}{c}{ Factors } \\
& A & B & C \\
\hline Number of defining variables (n) & 8 & 2 & 1 \\
Composite reliability score & 0.97 & 0.889 & 0.8 \\
Standard error of factor scores & 0.174 & 0.333 & 0.447 \\
& & & \\
\hline
\end{tabular}

1 
Table 4(on next page)

Statements showing agreement and disagreement across factors 


\begin{tabular}{|c|c|c|c|c|}
\hline \multirow{2}{*}{\multicolumn{2}{|c|}{$\frac{\text { No. }}{\text { Disagreement across factors }}$}} & \multicolumn{3}{|c|}{ Factor Score } \\
\hline & & \multirow{2}{*}{$\begin{array}{l}\mathbf{A} \\
-1\end{array}$} & \multirow{2}{*}{ B } & \multirow{2}{*}{$\begin{array}{l}\text { C } \\
0\end{array}$} \\
\hline 4 & $\begin{array}{l}\text { Further research comparing the methods and results of rapid reviews and systematic reviews is } \\
\text { required before I decide how I feel about rapid reviews. }\end{array}$ & & & \\
\hline 3 & $\begin{array}{l}\text { Deviating from accepted systematic review methods may introduce bias and impact the } \\
\text { validity of the resulting rapid review, which may be an unacceptable risk for some for } \\
\text { knowledge users. }\end{array}$ & 0 & 3 & 1 \\
\hline 2 & $\begin{array}{l}\text { When time allows, a comprehensive systematic review of all available evidence should always } \\
\text { be conducted. }\end{array}$ & -1 & 2 & -3 \\
\hline 47 & A rapid review cannot be a systematic review. & -2 & 3 & -2 \\
\hline 28 & $\begin{array}{l}\text { Knowledge users don't always need all of the evidence, they just need the best evidence to } \\
\text { support their decision, and what is 'best evidence' is specific to the knowledge user. }\end{array}$ & 1 & -2 & 3 \\
\hline \multicolumn{5}{|c|}{ Agreement across factors } \\
\hline 6 & Rapid reviews mean different things to different people. & 1 & 2 & 1 \\
\hline 7 & Rapid reviews should only precede a more comprehensive and rigorous systematic review. & -3 & -3 & -3 \\
\hline 34 & It is appropriate to endeavor to define a single, unique methodology for rapid reviews. & -1 & -1 & -1 \\
\hline 42 & Any review of evidence that takes longer than 3 months to produce is not a rapid review. & -1 & -2 & -1 \\
\hline 43 & Any review of evidence that takes longer than 1 month to produce is not a rapid review. & -2 & -1 & -2 \\
\hline
\end{tabular}


Figure 1

Steps in Q Methodology 


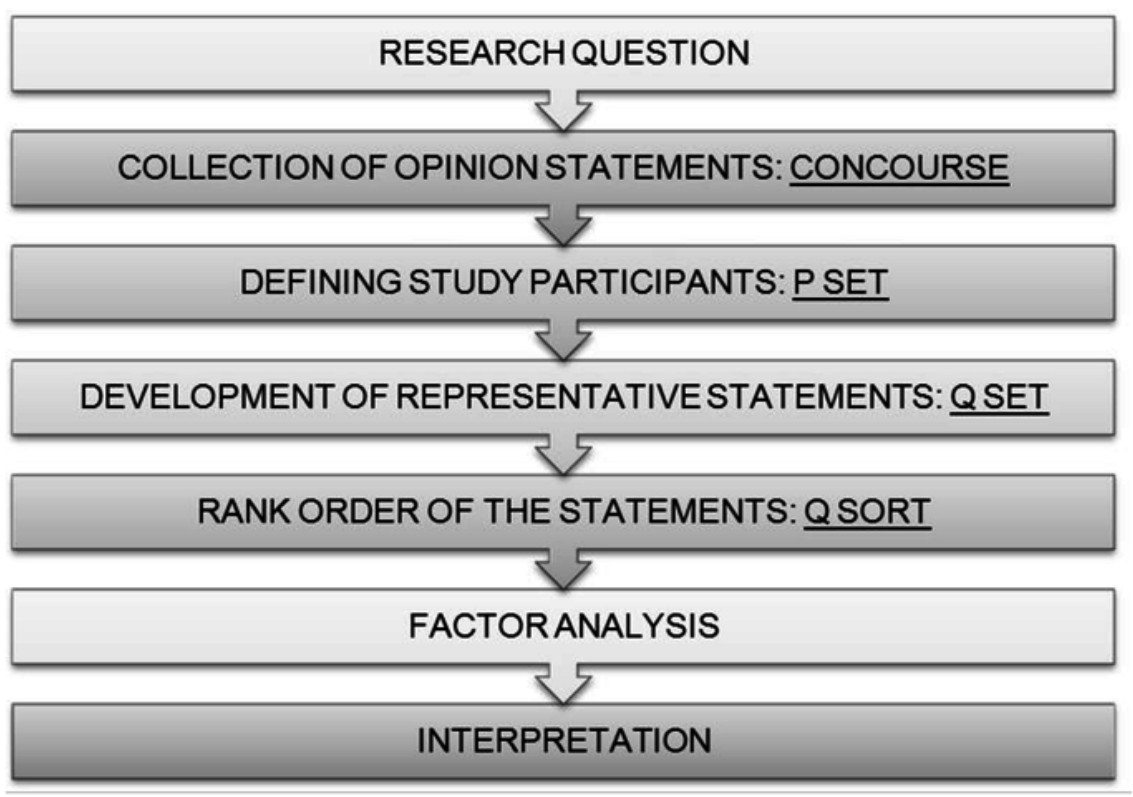


Figure 2

Fixed distribution for the Q-set

\begin{tabular}{|c|c|c|c|c|c|c|}
\hline Least Agree & \multicolumn{3}{c}{ Most Agree } \\
Statement Scores \\
-3 & -2 & -1 & 0 & 1 & 2 & 3 \\
\hline$(2)$ & $(6)$ & $(10)$ & $(14)$ & $(10)$ & $(6)$ & $(2)$ \\
\hline
\end{tabular}

